\title{
Detecting the First Quasars with ALMA
}

\author{
Dominik R. G. Schleicher ${ }^{1,2,3}$, Marco Spaans ${ }^{4}$, and Ralf S. Klessen ${ }^{3}$ \\ ${ }^{1}$ Leiden Observatory, P.O. Box 9513, NL-2300 RA Leiden, the Netherlands \\ Email: schleicher@strw.leidenuniv.nl \\ ${ }^{2}$ ESO Garching,Karl-Schwarzschild-Str. 2, 85748 Garching bei München, Germany \\ ${ }^{3}$ Zentrum für Astronomie der Universität Heidelberg, Institut für Theoretische Astrophysik, \\ Albert-Ueberle-Str. 2, 69120 Heidelberg, Germany. \\ ${ }^{4}$ Kapteyn Astronomical Institute, University of Groningen, P.O. Box 800, 9700 AV, \\ Groningen, the Netherlands \\ Email: spaans@astro.rug.nl
}

Keywords. astrochemistry, telescopes, galaxies: active, galaxies: high-redshift, galaxies: ISM

We show that ALMA is the first telescope that can probe the dust-obscured central region of quasars at $z>5$ with a maximum resolution of $\sim 30 \mathrm{pc}$ employing the $18 \mathrm{~km}$ baseline.

We explore the possibility of detecting the first quasars with ALMA (Schleicher, Spaans, $\&$ Klessen 2009). For this purpose, we adopt the Seyfert 2 galaxy NGC 1068 as a reference system and calculate the expected fluxes if this galaxy were placed at high redshift. This choice is motivated by the detailed observations available for this system and the absence of any indication for an evolution in metallicity in high-redshift quasars. It is a conservative choice due to the moderate column densities in NGC 1068, leading to moderate fluxes.

On large scales, the dominant excitation mechanism is soft-UV photons produced by the starburst, leading to strong emission in the $[\mathrm{C}$ II] $\lambda 158 \mu \mathrm{m}$ and the $[\mathrm{O} \mathrm{I}] \lambda 63 \mu \mathrm{m}$ line. Due to its unprecedented angular resolution, ALMA can probe the central $200 \mathrm{pc}$ of high-redshift quasars. Calculations using the X-ray dominated region code of Meijerink \& Spaans (2005) show that strong emission in the high- $J$ CO lines can be expected in these regions (see Figure 1). As these lines are redshifted into the ALMA bands, this may provide the first opportunity to detect these lines.

\section{References}

Meijerink, R. \& Spaans, M. 2005, A\&A, 436, 397

Schleicher, D. R. G., Spaans, M., \& Klessen, R. S. 2009, submitted to $A \& A$

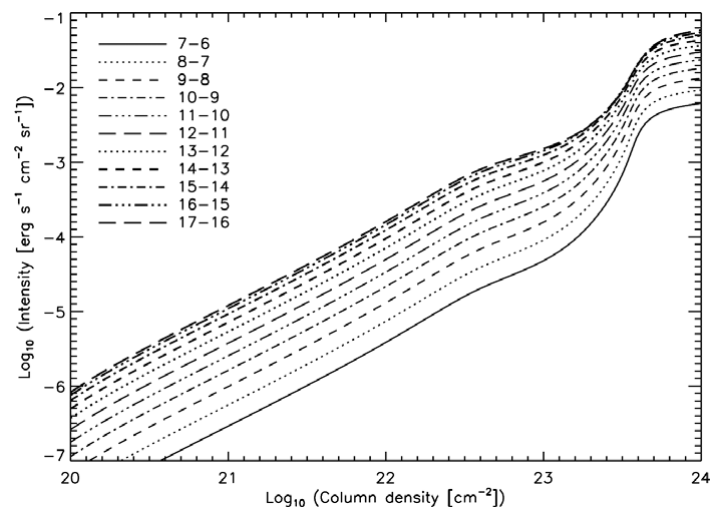

Figure 1. The expected intensities in the high- $J$ CO lines as a function of the cloud column density in the central region of a system like NGC 1068. 